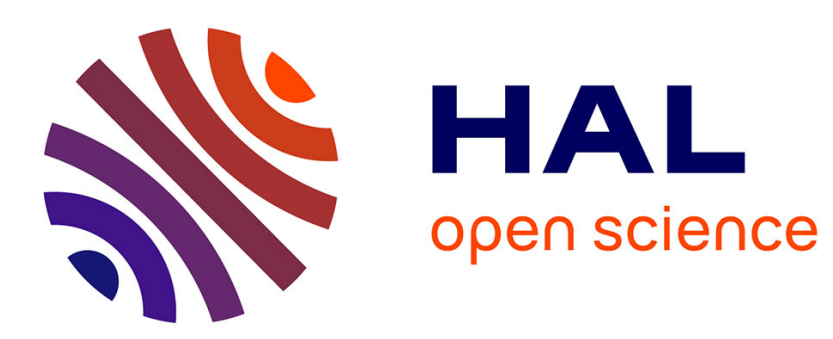

\title{
An Eulerian finite volume solver for multi-material fluid flows with cylindrical symmetry.
}

Aude Bernard-Champmartin, Jean-Philippe Braeunig, Jean-Michel Ghidaglia

\section{To cite this version:}

Aude Bernard-Champmartin, Jean-Philippe Braeunig, Jean-Michel Ghidaglia. An Eulerian finite volume solver for multi-material fluid flows with cylindrical symmetry.. Computers and Fluids, 2012, pp.7. 10.1016/j.compfluid.2012.09.014 . hal-00797200

\section{HAL Id: hal-00797200 \\ https://hal.science/hal-00797200}

Submitted on 6 Mar 2013

HAL is a multi-disciplinary open access archive for the deposit and dissemination of scientific research documents, whether they are published or not. The documents may come from teaching and research institutions in France or abroad, or from public or private research centers.
L'archive ouverte pluridisciplinaire HAL, est destinée au dépôt et à la diffusion de documents scientifiques de niveau recherche, publiés ou non, émanant des établissements d'enseignement et de recherche français ou étrangers, des laboratoires publics ou privés. 


\title{
An Eulerian Finite Volume solver for multi-material fluid flows with cylindrical symmetry
}

\author{
Aude Bernard-Champmartin ${ }^{\mathrm{a}, \mathrm{c}}$, Jean-Philippe Braeunig, ${ }^{\mathrm{b}, \mathrm{c}}$, Jean-Michel \\ Ghidaglia ${ }^{\mathrm{a}, \mathrm{c}}$ \\ ${ }^{a}$ CMLA, ENS CACHAN and CNRS UMR 8536, 61 avenue du Président Wilson, 94235 \\ CACHAN Cedex, France \\ ${ }^{b} C E A, D A M, D I F$, F-91297, Arpajon, France \\ ${ }^{c} L R C$ MESO, ENS CACHAN and CEA, DAM, DIF, 61 avenue du Président Wilson, \\ 94235 CACHAN Cedex, France
}

\begin{abstract}
In this paper, we adapt a pre-existing $2 D$ cartesian cell centered finite volume solver to treat the compressible $3 D$ Euler equations with cylindrical symmetry. We then extend it to multi-material flows. Assuming cylindrical symmetry with respect to the $\mathrm{z}$ axis (i.e. all the functions do not depend explicitly on the angular variable $\theta$ ), we obtain a set of 5 conservation laws with source terms that can be decoupled in 2 systems solved on a $2 D$ orthogonal mesh in which a cell as a torus geometry. A specific upwinding treatment of the source term is required and implemented for the stationary case. Test cases will be presented for vanishing and non-vanishing azimuthal velocity $u_{\theta}$.
\end{abstract}

Keywords: Finite Volumes, Axisymmetric Compressible Euler Equations, Multi-material flows

\section{The $3 D$ axisymmetric Euler equations}

In Braeunig et al. [5], a Eulerian Finite Volume solver was built for the computation of $1 D, 2 D$ and $3 D$ multi-material flows obeying to the compressible Euler equations. This solver was an extension to multi-material flows with sharp interfaces of the Finite Volume with Characteristic Flux 
(FVCF) solver ${ }^{1}$ (Ghidaglia et al. [7]) designed for single fluid or multi-fluid without sharp interfaces. In this paper we address the case of $2 D$ axisymmetric flows, that is to say with a symmetry around the z-axis.

We propose a quite general strategy. Starting with a cell-centered $2 D$ code that solves the Compressible Euler equations, we show how to build a $2 D$ code with cylindrical symmetry that uses the numerical fluxes of the $2 D$ cartesian code.

\subsection{Main differences between $2 D$ and $3 D$ axisymmetric Euler equations}

The $3 D$ Euler equations in cylindrical coordinates $(r, \theta, z)$ written in "conservative form" (i.e. $\frac{\partial}{\partial t} \bullet+\frac{1}{r} \frac{\partial}{\partial r} \bullet+\frac{\partial}{\partial z} \bullet$ ) reads:

$$
\begin{array}{r}
\frac{\partial \rho}{\partial t}+\frac{1}{r} \frac{\partial}{\partial r}\left(r \rho u_{r}\right)+\frac{\partial}{\partial z}\left(\rho u_{z}\right)=0, \\
\frac{\partial\left(\rho u_{r}\right)}{\partial t}+\frac{1}{r} \frac{\partial}{\partial r}\left(r\left(\rho u_{r}^{2}+p\right)\right)+\frac{\partial}{\partial z}\left(\rho u_{r} u_{z}\right)=\frac{1}{r}\left(\rho u_{\theta}^{2}+p\right), \\
\frac{\partial\left(\rho u_{z}\right)}{\partial t}+\frac{1}{r} \frac{\partial}{\partial r}\left(r\left(\rho u_{r} u_{z}\right)\right)+\frac{\partial}{\partial z}\left(\rho u_{z}^{2}+p\right)=0, \\
\frac{\partial\left(\rho u_{\theta}\right)}{\partial t}+\frac{1}{r} \frac{\partial}{\partial r}\left(r\left(\rho u_{r} u_{\theta}\right)\right)+\frac{\partial}{\partial z}\left(\rho u_{\theta} u_{z}\right)=-\frac{1}{r} \rho u_{\theta} u_{r}, \\
\frac{\partial(\rho E)}{\partial t}+\frac{1}{r} \frac{\partial}{\partial r}\left(r \rho u_{r}\left(E+\frac{p}{\rho}\right)\right)+\frac{\partial}{\partial z}\left(\rho u_{z}\left(E+\frac{p}{\rho}\right)\right)=0,
\end{array}
$$

where $E$ is the sum of the specific internal energy $e$ and the specific kinetic energy $\frac{1}{2}|u|^{2}$. This system is classically closed thanks to an equation of state: $\operatorname{EOS}(p, \rho, e)=0$. We consider also the case of swirling flows (see e.g. [6]) that corresponds to $u_{\theta} \neq 0$. Applications for such flows will be addressed elsewhere. Note that compared to the $2 D$ Euler equations we have 3 components for the velocity instead of 2. In Section 1.2 we will show how we recover a partial system with only 2 components for the velocity.

Since the system is expressed in the mobile cylindric basis $\left(e_{r}, e_{\theta}, e_{z}\right)$, source terms appear in equations (2) and (4). They can be treated either as centered terms ( $c f$. Sec. 2.1) or following an upwinding procedure (cf. Sec. 2.2) depending on the foreseen applications.

\footnotetext{
${ }^{1}$ The FVCF solver uses an approximate Riemann solver to calculate the fluxes, generalizing the Roe's method [12].
} 
Finally, observing that a rectangular cell has a torus geometry obtained by revolution around the $r=0$ axis $^{2}$, surfaces and volumes will depend explicitly on the radial coordinate $r$, affecting the numerical scheme.

\subsection{Decoupling of the $3 D$ axisymmetric Euler equations}

To adapt our $3 D$ problem with cylindrical symmetry to the $2 D$ code, we extract the velocity $u_{\theta}$ from the previous system by defining a partial specific energy $E_{s}$ :

$$
E_{s}=E-\frac{1}{2}\left|u_{\theta}\right|^{2}=e+\frac{1}{2}\left|u_{r}\right|^{2}+\frac{1}{2}\left|u_{z}\right|^{2},
$$

thus independent of $u_{\theta}$. Doing so the velocities $u_{r}$ and $u_{z}$ can be handled in the same manner as $2 D$ plan velocities.

Then, combining equations (3) and (5), we obtain the equation for the specific partial total energy:

$$
\frac{\partial\left(\rho E_{s}\right)}{\partial t}+\frac{1}{r} \frac{\partial}{\partial r}\left(r \rho u_{r}\left(E_{s}+\frac{p}{\rho}\right)\right)+\frac{\partial}{\partial z}\left(\rho u_{z}\left(E_{s}+\frac{p}{\rho}\right)\right)=\frac{1}{r} \rho\left(u_{\theta}\right)^{2} u_{r} .
$$

We can now isolate a four equations system, for density, $u_{r}, u_{z}$ and partial energy $E_{s}$, which has the same number of conservative variables as in the $2 \mathrm{D}$ cartesian case:

$$
\frac{\partial V}{\partial t}+\frac{1}{r} \frac{\partial}{\partial r} F_{r}(V)+\frac{\partial}{\partial z} F_{z}(V)=G(V)
$$

with:

$$
\begin{gathered}
V=\left(\begin{array}{c}
\rho \\
\rho u_{r} \\
\rho u_{z} \\
\rho E_{s}
\end{array}\right), \quad G(V)=\left(\begin{array}{c}
0 \\
\frac{1}{r}\left(\rho u_{\theta}^{2}+p\right) \\
0 \\
\frac{\rho}{r}\left|u_{\theta}\right|^{2} u_{r}
\end{array}\right), \\
F_{r}(V)=\left(\begin{array}{c}
r \rho u_{r} \\
r\left(\rho u_{r}^{2}+p\right) \\
r \rho u_{z} u_{r} \\
r \rho u_{r}\left(E_{s}+\frac{p}{\rho}\right)
\end{array}\right), \quad F_{z}(V)=\left(\begin{array}{c}
\rho u_{z} \\
\rho u_{r} u_{z} \\
\rho u_{z}^{2}+p \\
\rho u_{z}\left(E_{s}+\frac{p}{\rho}\right)
\end{array}\right) .
\end{gathered}
$$

\footnotetext{
${ }^{2}$ except for the the cells containing the axis $r=0$ which have cylindrical geometry.
} 
In that system, the velocity $u_{\theta}$ only appears in the source term $G(V)$. We close this system by using (4) which is the advection of the velocity $u_{\theta}$ and source terms.

\section{Extension of the $2 D$ FVCF single material scheme to the ax- isymmetric case:}

In the axisymmetric case, each cell of the $2 D$ uniform orthogonal grid represents the axisymmetric volume obtained by revolution of the rectangle around the $r=0$ axis ( $c f$. Fig. 1). In cylindrical coordinates, a cell $K_{i j}$ of the mesh is then defined by:

$$
K_{i j}=\left\{(r, z, \theta), r_{i-1 / 2} \leq r \leq r_{i+1 / 2}, z_{j-1 / 2} \leq z \leq z_{j+1 / 2}, 0 \leq \theta \leq 2 \pi\right\} .
$$

The surface or the volume of a cell then depends on the radial position.

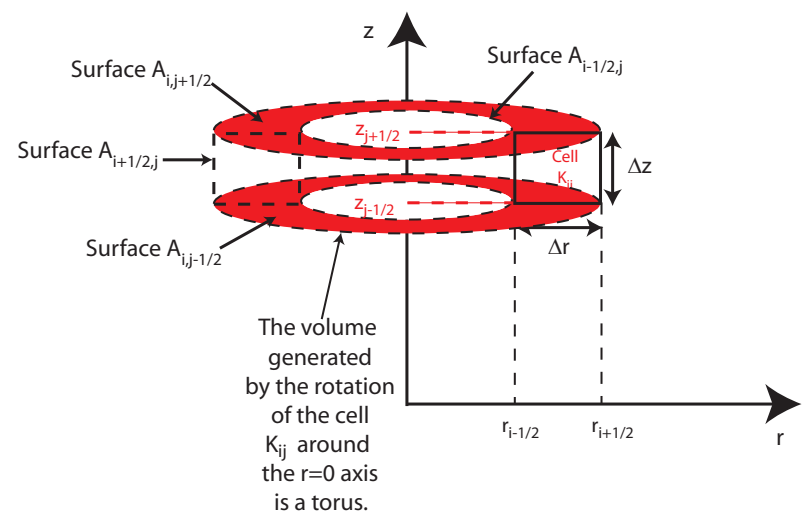

Figure 1: The $2 D$ cell $K_{i j}$ represents a torus described by the revolution around $r=0$ axis.

Denoting by $\left|K_{i j}\right|$ the volume of the cell $K_{i j}, A_{i+1 / 2, j}$ (resp. $\left.A_{i-1 / 2, j}\right)$ the outer (resp. inner) surface of the torus and $A_{i, j+1 / 2}$ (resp. $A_{i, j-1 / 2}$ ) the upper (resp. the downer) surface of the torus, we have:

$$
\begin{array}{r}
\left|K_{i j}\right|=\pi \Delta z\left(r_{i+1 / 2}^{2}-r_{i-1 / 2}^{2}\right)=\underbrace{\Delta z \Delta r}_{2 D \text { volume }} \pi \underbrace{\left(r_{i+1 / 2}+r_{i-1 / 2}\right)}_{\equiv 2 r_{i}}, \\
A_{i \pm 1 / 2}=2 \pi r_{i \pm 1 / 2} \Delta z, \quad A_{i, j \pm 1 / 2}=\pi\left(r_{i+1 / 2}^{2}-r_{i-1 / 2}^{2}\right) .
\end{array}
$$


Note that the ratio between the surfaces of perpendicular to $r$ faces and the volume of the torus is not constant whereas it remains constant for the surfaces of perpendicular to $z$ faces:

$$
\frac{A_{i \pm 1 / 2, j}}{\left|K_{i j}\right|}=\frac{r_{i \pm 1 / 2}}{r_{i}} \frac{1}{\Delta r}, \quad \frac{A_{i, j \pm 1 / 2}}{\left|K_{i j}\right|}=\frac{1}{\Delta z} .
$$

The finite volume scheme is deduced from the system of equations (8) by integrating it on a control volume $K_{i j}$. Setting $V_{i j}^{n}=\frac{1}{\left|K_{i j}\right|} \int_{K_{i j}} V\left(x, t^{n}\right) d \tau$, we get:

$$
V_{i j}^{n+1}=V_{i j}^{n}-\frac{2 \pi \Delta t}{\left|K_{i j}\right|} \int_{\partial K_{i j}} \underbrace{\left(\left(\begin{array}{c}
F_{r}(V) \\
r F_{z}(V)
\end{array}\right) \cdot \mathbf{n}\right)^{t}}_{r \text {.(fluxes of the } 2 D \text { Euler equations) }} d r d z+\frac{\Delta t}{r_{i}} \sum_{i j}^{n},
$$

where $\Sigma_{i j}^{n}$ approximate the quantity: $\sum_{i j}^{n} \sim \frac{r_{i}}{\left|K_{i j}\right|} \int_{K_{i j}} G(V) d \tau$ and $\mathbf{n}$ the outgoing normal.

We remark that the fluxes $\frac{1}{r} F_{r}(V)=F_{2 D}(V) \cdot n_{r}\left(\right.$ resp. $F_{z}(V)=F_{2 D}(V)$. $n_{z}$ ) correspond exactly to the $2 D$ fluxes of the $2 D$ cartesian Euler equations on horizontal sides (resp. vertical sides) of the cell. Indeed the $2 D$ fluxes write (cf. e.g. [4])

$$
F_{2 D}(V) \cdot \mathbf{n}=(\rho(\mathbf{u} \cdot \mathbf{n}), \rho \mathbf{u}(\mathbf{u} \cdot n)+p \cdot \mathbf{n},(\rho E+p)(\mathbf{u} \cdot \mathbf{n}))^{t},
$$

where $\mathbf{n} \in \mathbb{R}^{2}$ is a unitary vector and in our case we take $\mathbf{u}=\left(u_{r}, u_{z}\right)^{t}$ and $E$ is replaced by $E_{s}$. Using this comment, we replace now in (15) the axisymmetric fluxes $\frac{1}{r} F_{r}(V)$ and $F_{z}(V)$ by the $2 D$ FVCF numerical fluxes $f_{i \pm 1 / 2, j}$ (left and right fluxes), $f_{i, j \pm 1 / 2}$ (up and down fluxes) ( $c f$. [7] for the exact expression of these fluxes composed of an average of $2 D$ fluxes in neighboring cells plus an upwinding flux), and we get:

$$
\begin{aligned}
V_{i j}^{n+1} & =V_{i j}^{n}-\frac{\Delta t}{\Delta r}\left[\frac{r_{i+1 / 2}}{r_{i}} f_{i+1 / 2, j}-\frac{r_{i-1 / 2}}{r_{i}} f_{i-1 / 2, j}\right] \\
& -\frac{\Delta t}{\Delta z}\left(f_{i, j+1 / 2}-f_{i, j-1 / 2}\right)+\frac{\Delta t}{r_{i}} \sum_{i j}^{n} .
\end{aligned}
$$

Concerning the advection along $u_{\theta}$, we integrate the equation (4) on the control volume $K_{i j}$. Setting $v_{\theta_{i j}}^{n}=\frac{1}{\left|K_{i j}\right|} \int_{K_{i j}} v_{\theta}\left(x, t^{n}\right) d \tau$, where $v_{\theta}=\rho u_{\theta}$, we 
obtain:

$$
\begin{aligned}
v_{\theta_{i j}}^{n+1}= & v_{\theta_{i j}}^{n}-\frac{\Delta t}{\Delta r}\left[\frac{r_{i+1 / 2}}{r_{i}} f_{\theta_{i+1 / 2, j}}-\frac{r_{i-1 / 2}}{r_{i}} f_{\theta_{i-1 / 2, j}}\right] \\
& -\frac{\Delta t}{\Delta z}\left(f_{\theta_{i, j+1 / 2}}-f_{\theta_{i, j-1 / 2}}\right)-\frac{\Delta t}{r_{i}} \Sigma_{\theta_{i j}},
\end{aligned}
$$

where $\Sigma_{\theta_{i j}}$ approximate the quantity

$$
\Sigma_{\theta_{i j}} \sim \frac{r_{i}}{\left|K_{i j}\right|} \int_{K_{i j}} \rho u_{\theta} u_{r} d r d z
$$

and the fluxes $f_{\theta_{i \pm 1 / 2, j}}$ and $f_{\theta_{i, j \pm 1 / 2}}$ are simply given by an upwind procedure.

We observe that the numerical scheme (17) differs only from the $2 D$ cartesian scheme by the presence of the ratio $\frac{r_{i \pm 1 / 2}}{r_{i}}$ in front of the numerical flux along the $\mathrm{r}$ axis. We recover here the difference between the ratio of the surface of an edge over the volume of the cell: this ratio is constant in the $2 D$ cartesian case $(1 / \Delta r$ or $1 / \Delta z)$ whereas in the axisymmetric case, it depends on the position along the $r$ axis $\left(\frac{1}{\Delta r} \frac{r_{i \pm 1 / 2}}{r_{i}}\right)$ but remains constant along the $z$ axis. Furthermore a geometrical source term also appears in equation (17). It will be discretized following two different ways, described in the next sections. We point out that the extension's procedure from the $2 D$ case to the $2 D$ with cylindrical symmetry one does not modify the precision of the original scheme. Here, we present the order 1 version of the scheme. Using MUSCL techniques [8] combining with a Strang splitting [13], one can easily extend the results to a second order scheme.

\subsection{Centered discretization of the source term:}

The first possibility is to approximate the integrand of the source term by its centered value inside each cell and we obtain for $\sum_{i j}^{n}$ :

$$
\Sigma_{i j}^{n}=\left(\begin{array}{c}
0 \\
\left(\rho u_{\theta}^{2}+p\right)_{i j}^{n} \\
0 \\
\left(\rho u_{\theta}^{2} u_{r}\right)_{i j}^{n}
\end{array}\right)
$$

and for $\sum_{\theta_{i, j}}^{n}$ :

$$
\Sigma_{\theta_{i, j}}^{n}=-\left(\rho u_{\theta} u_{r}\right)_{i j}^{n} .
$$


Thus the two numerical schemes (17) for $V$ and (18) for $V_{\theta}$ read with a centered source term:

$$
\begin{aligned}
V_{i j}^{n+1}=V_{i j}^{n}-\frac{\Delta t}{\Delta r} & {\left[\frac{r_{i+1 / 2}}{r_{i}}\left(f_{i+1 / 2, j}-\sum_{i j}^{n}\right)-\frac{r_{i-1 / 2}}{r_{i}}\left(f_{i-1 / 2, j}-\sum_{i j}^{n}\right)\right] } \\
& -\frac{\Delta t}{\Delta z}\left(f_{i, j+1 / 2}-f_{i, j-1 / 2}\right) . \\
v_{\theta_{i j}}^{n+1}= & v_{\theta_{i j}}^{n}-\frac{\Delta t}{\Delta r}\left(\frac{r_{i+1 / 2}}{r_{i}}\left(f_{\theta_{i+1 / 2, j}}-\Sigma_{\theta_{i, j}}^{n}\right)-\frac{r_{i-1 / 2}}{r_{i}}\left(f_{\theta_{i-1 / 2, j}}-\Sigma_{\theta_{i, j}}^{n}\right)\right) \\
- & \frac{\Delta t}{\Delta z}\left(f_{\theta_{i, j+1 / 2}}-f_{\theta_{i, j-1 / 2}}\right) .
\end{aligned}
$$

Note that the source terms only exist for the r-axis direction and keep the scheme along the z-axis direction unchanged with respect to the cartesian case.

\subsection{Upwinding of the source term:}

For stationary solutions computation, it is well known that numerical fluxes obtained by upwinding induces a bias if the source term is discretized with a centred formula (see e.g. Roe [11]). Following Alouges et al. [1], we have modified (20) and (21) in order to properly capture stationary solutions (details are given in the technical report Bernard-Champmartin et al. [2]). Note that the decentering of the source term, allowing the conservation of the stationary solution leads necessary to a first order approximation of it instead of the second order that would be obtained with a centered one.

\subsection{Extension to the two materials case}

The method presented above is for the one material case. We have also extended the $2 D$ FVCF-NIP method ([4],[5]) (which is an extension of the original FVCF scheme which deals with two materials flows with sharp interface capturing) in the $3 D$ case with cylindrical symmetry. Details are given in the technical report Bernard-Champmartin et al. [2] and the numerical results presented in the following section (Sec. 3) are performed with the two materials version of the code. 


\section{Numerical simulations:}

In this section, some numerical test cases are presented using our modified $2 D$ axisymmetric code. The aim is to validate the modifications performed in order to take into account the axisymmetric case on exact solutions, such as simple advection test cases in Sec. 3.1 or as a stationary solution of the $3 D$ Euler equation with cylindrical symmetry when the azimuthal velocity $u_{\theta}$ is taken into account. The main interest of having a $2 D$ code with cylindrical symmetry, besides being able to consider 3 components of velocity, is to have the ability to conserve the initial shape of the case and it is the object of the third case of the Sec. 3.1. Study of the precision of the scheme has already been done for the $2 D$ version and can be found in previous articles $[5,3,9]$.

\subsection{Cases with $u_{\theta}=0$ (non-swirling flow).}

First, we check the simple case of a density discontinuity advection at velocity $u_{r}$ and then $u_{z}$ to validate the modifications performed in the code to extend it to the $2 D$ case with axisymmetric geometry.

The first case present the advection at a velocity $u_{r}$ (all the quantities depend only on $r)$. The pressure is constant in the whole domain $\left(p=p_{0}=1\right)$ and checks a perfect gas law with $\gamma=7 / 5$. The domain of simulation is from $r=0$ to $r=R$ (here $R=30$ ) for the $r$ direction. The velocity $u_{r}$ takes the value $0.1 \mathrm{~m} . \mathrm{s}^{-1}$ in the domain $[R / 3,2 R / 3]$ and is zero elsewhere (the others components of the velocity are set to zero). The density discontinuity is put at $r=\frac{R}{2}: \rho=\rho_{0} \frac{R}{3 r}$ for $r \in\left[\frac{R}{3}, \frac{R}{2}\right]$ and $\rho=4 \rho_{0} \frac{R}{3 r}$ for $r \in\left[\frac{R}{2}, \frac{2 R}{3}\right]$ and extended by continuity to a constant value for the rest of the domain ( $\rho_{0}$ is taken to 25). In Fig. 2, the density discontinuity is well advected along the $r$ direction at the velocity $u_{r}=0.1 \mathrm{~m} . \mathrm{s}^{-1}$. 


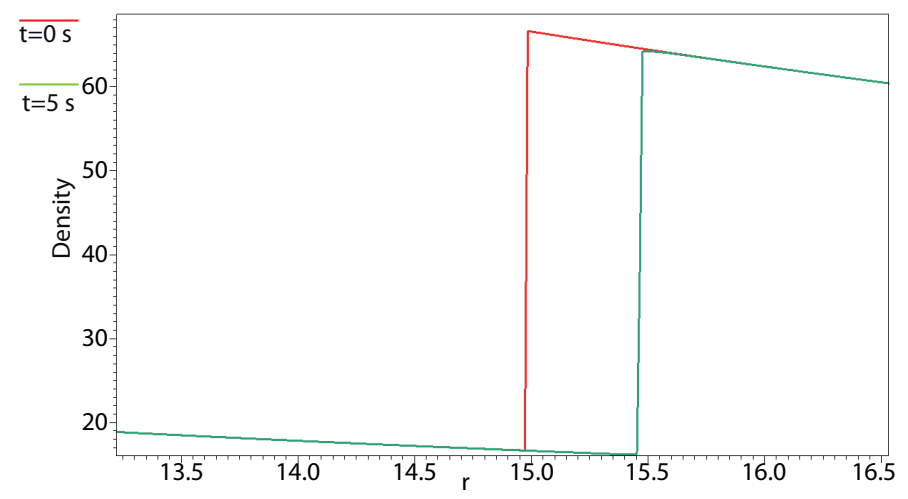

Figure 2: Advection at the velocity $u_{r}=0.1 \mathrm{~m} \cdot \mathrm{s}^{-1}$ of a discontinuous density $\rho$ (all the quantities depend only on $r$ ). We represent in red the initial density around the zone of discontinuity $r=\frac{R}{2}$ for the density and in green the result at $t=5 \mathrm{~s}\left(N_{x}=2500\right.$ for the whole domaine $r \in[0,30])$.

Then, we consider the advection along the $z$ axis of a square shaped material of density $\rho=2$ and velocity $u_{z}=1 \mathrm{~m} \cdot \mathrm{s}^{-1}$ surrounded by an other material of density $\rho=1$ with still a perfect gaz law $(\gamma=1.4)(c f$. Fig. 3 and Fig. 4 for a $1 D$ cut of the density along the line $r=0.6$ ).

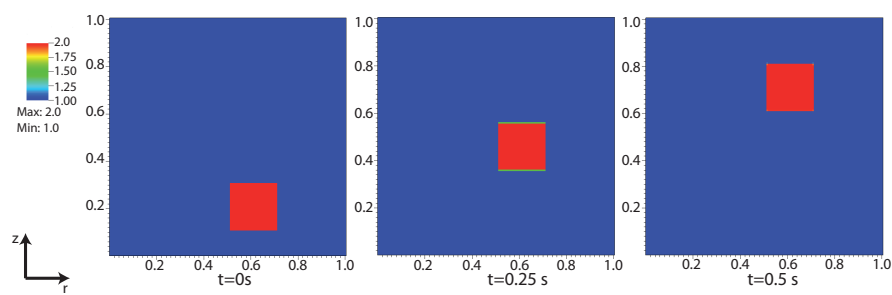

Figure 3: Advection at the velocity $u_{z}=1 \mathrm{~m} \cdot \mathrm{s}^{-1}$ of a square shaped material of density $\rho=2$ (in red for $t=0 \mathrm{~s}$ ). The square shape geometry uprise along the simulation.

We now look at the solution of the classical Sod test case extended for a spherical geometry (see ref. [10,6]): a half-circle (which represents a sphere of center $\left(r_{0}, z_{0}\right)=(0,1)$ and radius $R=\sqrt{r^{2}+z^{2}}=0.5$ generated by rotation around the $r=0$ axis) is placed side by side along the $r=0$ axis. The initialization of the domain is $(\rho, p, \vec{u})=(1,1, \overrightarrow{0})$ for the inner part of the 


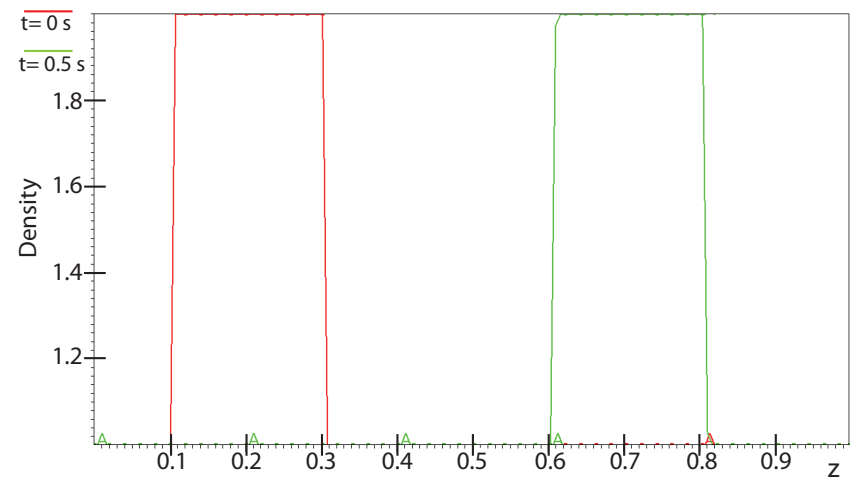

Figure 4: Advection at the velocity $u_{z}=1 \mathrm{~m} \cdot \mathrm{s}^{-1}$ of a square shaped material of density $\rho=2$ (in red for $t=0 \mathrm{~s}$ ). The cut is performed along the line $r=0.6$. At $t=0.5 \mathrm{~s}$ the square shaped geometry has moved of $0.5 \mathrm{~m}$ (curve in green).

circle and the outside is initialized with the value $(\rho, p, \vec{u})=(0.125,0.1, \overrightarrow{0})^{3}$. For that test case, the shape of the initial interface entertains non linear interfaces between materials, which are close to the direction of the axis especially at $r=0$ and $r=0.5)$. Results are obtained with the EnhancedNIP (ENIP) method([9]), which cures an inconsistency in the former method NIP [4], and shows good results.

We only present here the results obtained with the ENIP version of interface reconstruction extended for our $2 D$ axisymmetric code (Fig. 5).

\footnotetext{
${ }^{3}$ We simulate the whole half circle to free us from boundary conditions in mixed cells at $z=0$ axis. All the results we present in the following are zoomed on the upper quarter of the circle since the problem is totally symmetric.
} 


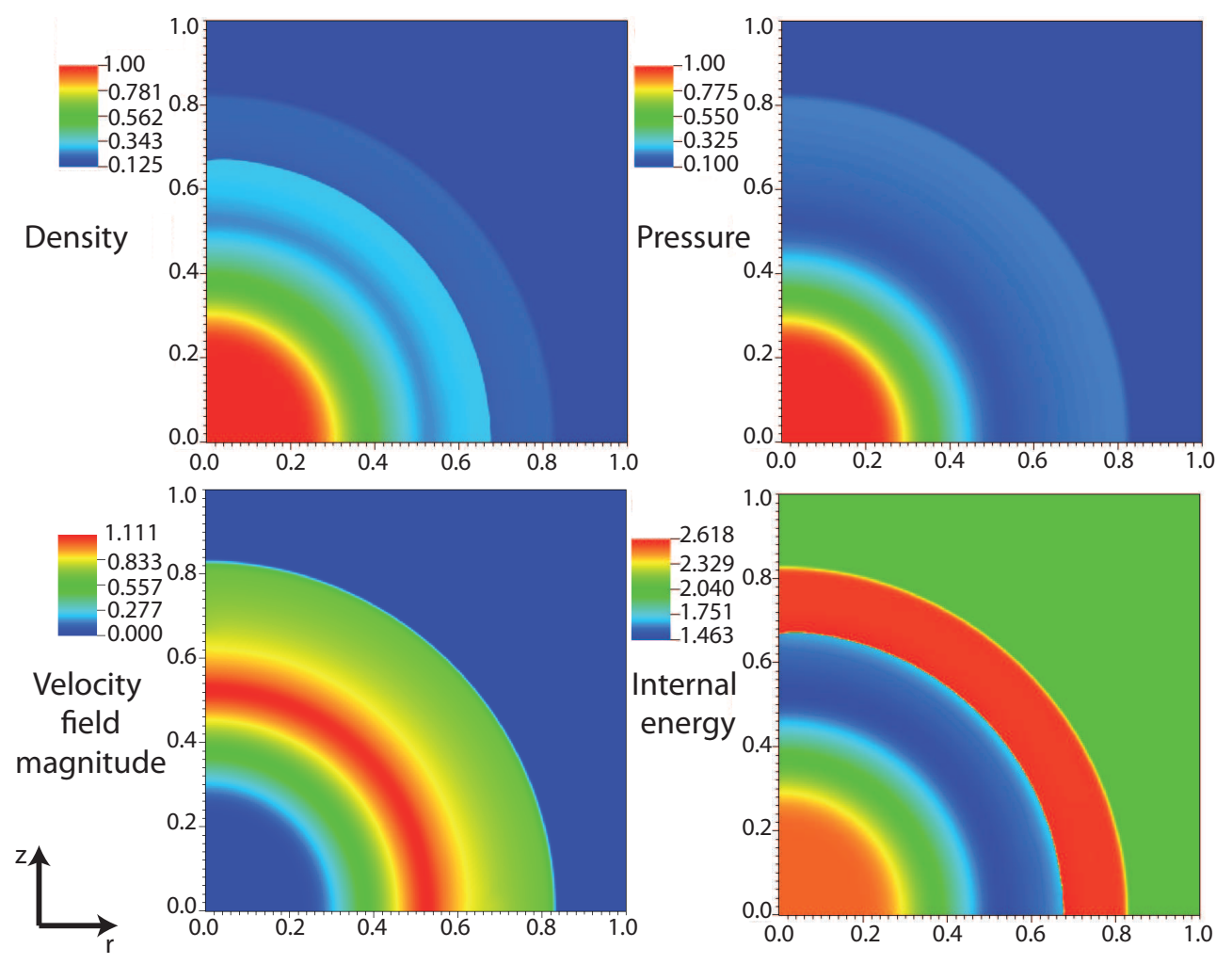

Figure 5: Spherical Sod test case (we represent here only the upper quarter of the half circle), $N_{r}=N_{z}=400$. We observe the results given by our $2 D$ axisymmetric code at $t=0.2 \mathrm{~s}$ for the density, the velocity field magnitude, the pressure and the internal energy with the ENIP version of interface reconstruction between materials. A slight default remains nevertheless present near the axis of symmetry.

We then perform $1 D$ line cuts of the $2 D$ results for all the variables with the angle $\theta=0$ (i.e. along the $z=0$ axis), then for $\theta=45$ (i.e. along the line $r=z$ ) and finally along the $r=0$ axis (cf. Fig. 6). 

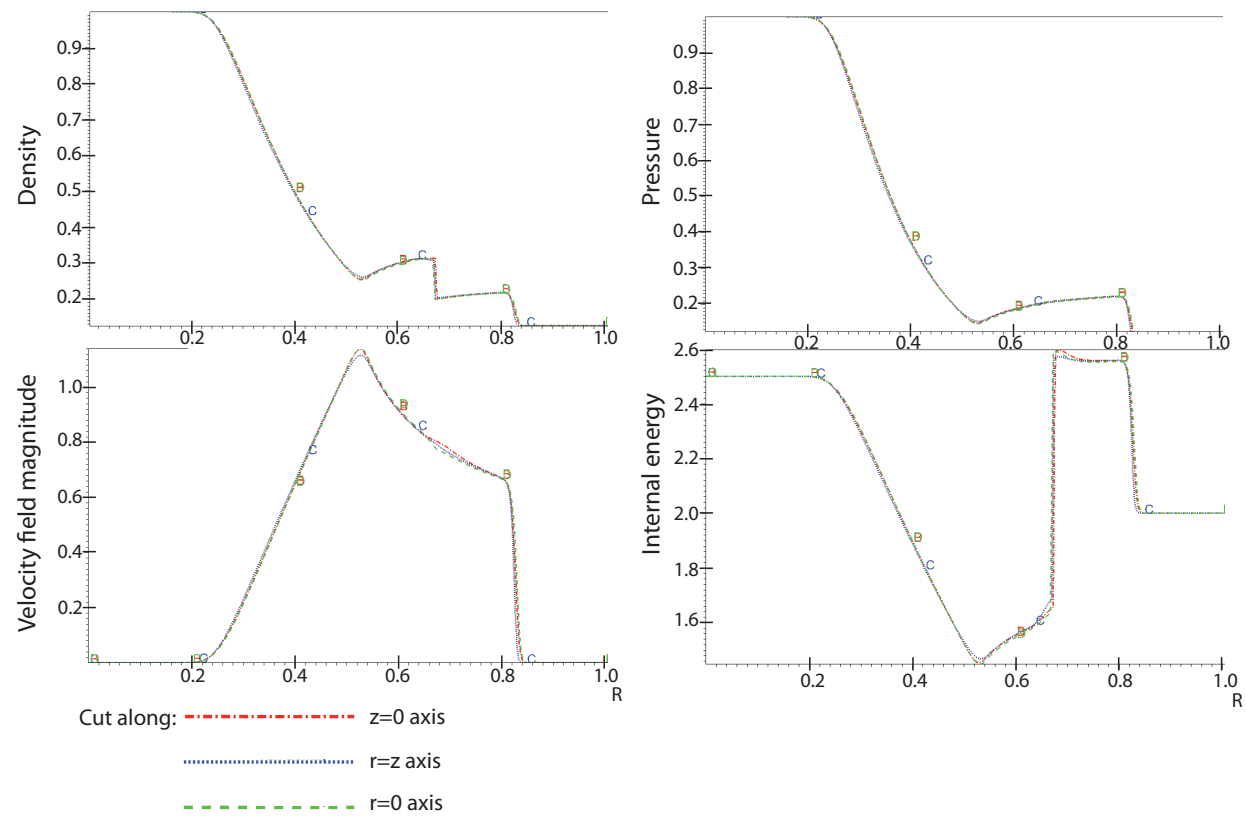

Figure 6: Spherical sod test case with ENIP interfaces reconstruction. We see that thanks to the ENIP axisymmetric version of the interface reconstruction, the sphericity conservation of the solutions is good: the cuts along the $r=0$ axis (in green) and along $z=0$ axis (in red) are very closed to the $r=z$ axis one.

A small defect of sphericity still existing close to the revolution axis $r=$ 0 and can be explained by the ratio of volume between two neighboring cells which is larger on the vicinity of this axis and also by the boundary conditions for mixed cells on the $r=0$ axis. Since no analytical solution of the spherical Sod shock test case is available, we compare the results given by our FVCF-2D Axi code with the results done with a $1 D$ version of the code with spherical symmetry, we present in Fig. (7) the cut along the $r=z$ axis and the results given by the $1 D$ code. 

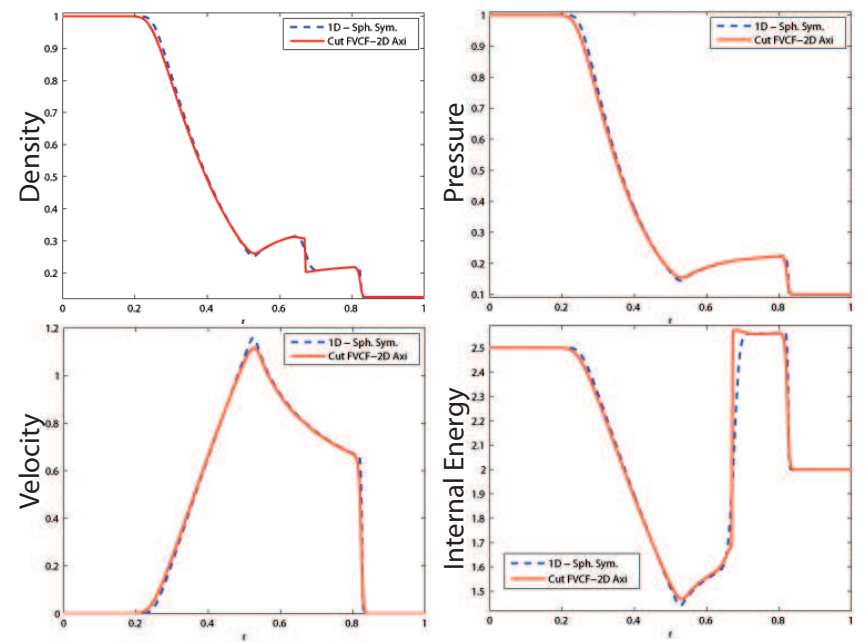

Figure 7: Profile of the solution at $t=0.2 \mathrm{~s}$ on the spherical Sod test case. Comparison is performed between a cut along the $r=z$ axis obtained by our FVCF-2D with cylindrical symmetry code with ENIP interfaces reconstruction (solid red line) and a $1 D$ version of the FVCF scheme with spherical terms (dotted blue line) $(N x=500)$. We observe the rarefaction wave, the shock and then the contact discontinuity. The contact discontinuity is well captured by the interface reconstruction when using the 2 material scheme. The results are in good agreement for all the quantities.

Finally, we plot in the Fig. 8 the error for the density, i.e. the absolute difference between our code with $N r=N z=500$ and a reference solution obtained with the $1 D$ version of the code with spherical symmetry performed with $N x=10000^{4}$.

\footnotetext{
${ }^{4}$ We are grateful to Sylvain Faure of the university Paris Sud for having given us the data of the $1 D$ with spherical symmetry Sod test case.
} 


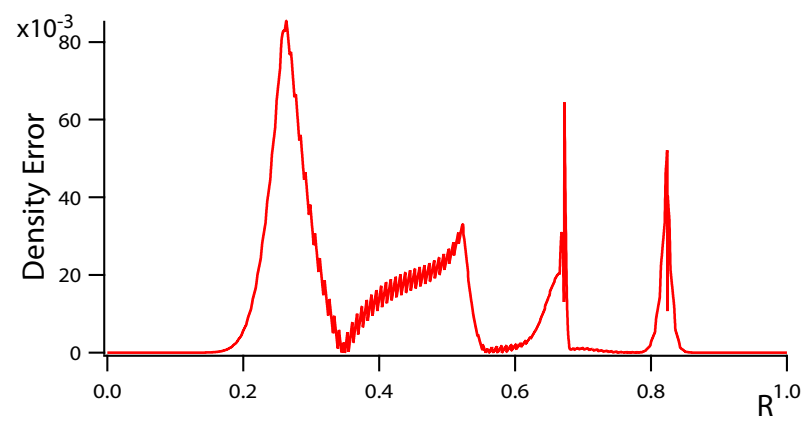

Figure 8: Curve of the absolute error of the density on the spherical Sod test case in function of the radius $R$ of the sphere. We plot the absolute difference between the converged solution (curve given by the $1 D$ with spherical symmetry code with $N x=10000$ ) and with our code (we took the cut along the $r=z$ axis) with $N r=N z=500$.

\subsection{Case with $u_{\theta}$ different of zero (swirling flows):}

In this section, we present one case with a velocity $u_{\theta}$. We consider a stationary solution of the axisymmetric Euler system (1)-(5) in a case where all the variables are only depending on $r$. For this case, an upwinding of the source term is needed ( $c f$. Sec. 2.2) and up to now the upwinding is available only for 1 material case.

The initial functions are defined by part:

$$
\overrightarrow{u_{r}}=0, \rho(r)=\rho_{\text {ext }} \varphi\left(r_{\text {int }}\right)^{\frac{1}{\gamma-1}}, p(r)=p_{\text {ext }} \varphi\left(r_{\text {int }}\right)^{\frac{\gamma}{\gamma-1}} \text {, for } 0 \leq r \leq r_{\text {int }} \text {, }
$$

and for $r>r_{\text {int }}$ :

$$
\begin{gathered}
u_{r}=0, u_{\theta}=f(r), u_{z}(r)=\frac{G(r)}{\rho_{\mathrm{ext}}} \varphi(r)^{-\frac{1}{\gamma-1}}, \rho(r)=\rho_{\mathrm{ext}} \varphi(r)^{\frac{1}{\gamma-1}}, \\
p(r)=p_{\mathrm{ext}} \varphi(r)^{\frac{\gamma}{\gamma-1}}, \quad \text { with } \varphi(r)=1-\frac{(\gamma-1) \rho_{\mathrm{ext}}}{\gamma p_{\mathrm{ext}}} \int_{r}^{r_{\mathrm{ext}}} \frac{f(s)^{2}}{s} d s
\end{gathered}
$$

and are solution of the system (1)-(5) with $r \in\left[0, r_{\text {ext }}\right]$. In the simulation, we take for the functions $f$ and $G$ : $f(r)=\frac{2}{r}$ and $G(r)=$ cst $=1.5$. The domain of simulation is $\left[0, r_{\text {ext }}\right]=[0,2]$ for the $r$ direction $\left(r_{\text {int }}=1, p_{\text {ext }}=2\right.$ and $\rho_{\text {ext }}=1$ for the simulation) ( $c f$. Fig. 9 and Fig. 10$)$. 


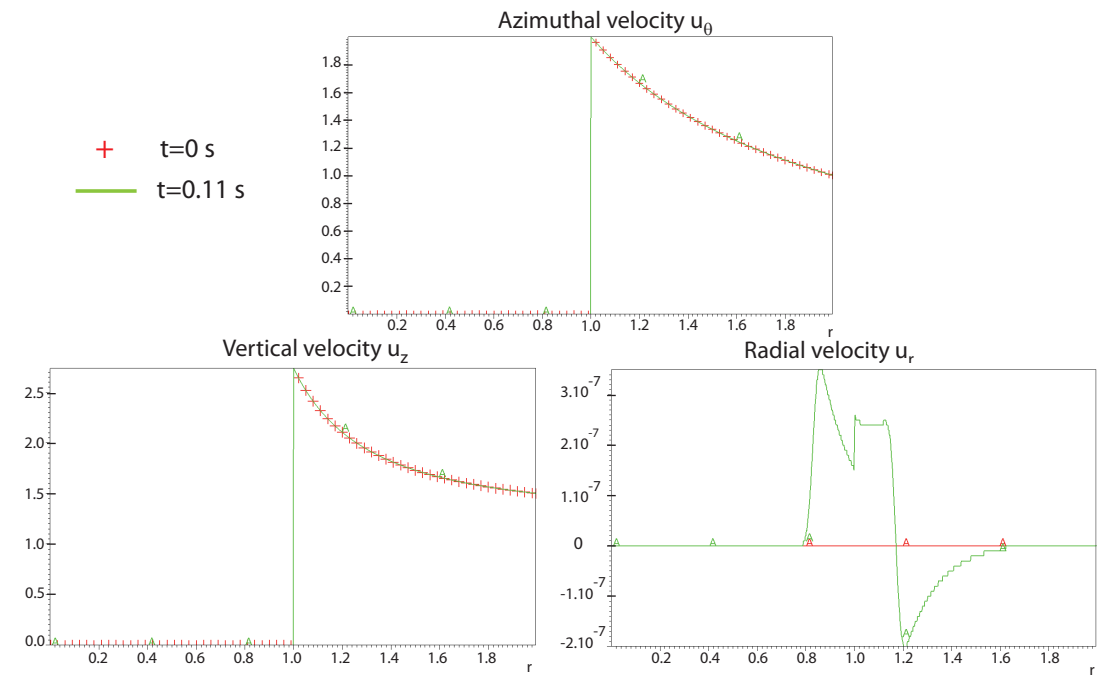

Figure 9: Stationary solution with a velocity $u_{\theta}$. For all the variables (we represent here only the velocity field), the initial solution is represented in red cross and the solution at $t=0.11 \mathrm{~s}$ in solid green line. We observe that all the variables remain constant along the simulation. The numerical scheme produce very small oscillations for the velocity $u_{r}$ $\left(10^{-7}\right.$ magnitude).

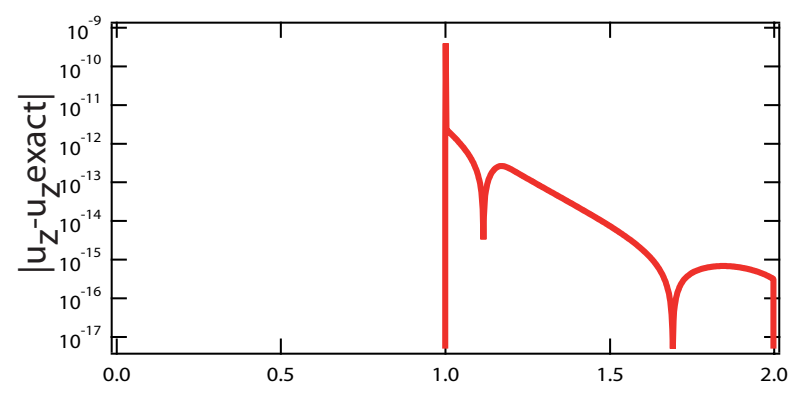

Figure 10: Stationary solution with a velocity $u_{\theta}$. Exemple of the absolute error (between the results of the code and the exact solution) of the velocity $u_{z}$ in semi-log scale. Although being first order, the scheme with a decentered source term capture with accuracy the stationary solution, the deviation remaining very small. 


\section{Conclusions}

We have extended a $2 D$ finite volume solver with cartesian grid for the compressible Euler equations in a $2 D-A x i$ code with symmetry around the $r=0$ axis. Dependance on the radial coordinate, source terms and a treatment on the third component of velocity $u_{\theta}$ has been added on the numerical scheme. We have validated our code on some test cases and in particular for the spherical Sod test case which allows us to check the sphericity conservation of the geometry.

All the work we performed are applied to the FVCF flux scheme solver [5] but the modifications performed in the code can be adapted at any other finite volume solver: Roe [12], Lax-Friedrichs...

Furthermore, it would be interesting to treat test cases with comparisons to experiments or to other codes and especially to check the behavior of $u_{\theta}$ in the simulations.

\section{References}

[1] F. Alouges, J.M. Ghidaglia, M. Tajchman, On the interaction of upwinding and forcing for nonlinear hyperbolic systems of conservation laws, Technical Report, Preprint of CMLA, http://www.cmla.enscachan.fr/documentation/prepublications/1999.html, 1999.

[2] A. Bernard-Champmartin, J.M. Ghidaglia, Equations d'Euler compressibles: extension d'un solveur volume fini 2D plan au cas 3D géométrie cylindrique., Preprint of CMLA 2011-12, CMLA, http://tinyurl.com/FVCFAXI, 2011.

[3] J. Braeunig, An algorithm to control the pressure evolution for the FVCF-NIP method for compressible multi-material fluid flows, Int. J. Finite Vol. 7 (2010) 101-132.

[4] J.P. Braeunig, Sur la simulation d'écoulements multi-matériaux par une méthode eulérienne directe avec capture d'interfaces en dimensions 1, 2 et 3., Ph.D. thesis, ENS Cachan, 2007.

[5] J.P. Braeunig, B. Desjardins, J.M. Ghidaglia, A totally Eulerian finite volume solver for multi-material fluid flows, Eur. J. Mech. B Fluids 28 (2009) 475-485. 
[6] S. Clain, D. Rochette, R. Touzani, A multislope MUSCL method on unstructured meshes applied to compressible Euler equations for axisymmetric swirling flows, J. Comput. Phys. 229 (2010) 4884-4906.

[7] J.M. Ghidaglia, A. Kumbaro, G.L. Coq, On the numerical solution to two fluid models via a cell centered finite volume method, European Journal of Mechanics - B/Fluids 20 (2001) 841 - 867.

[8] B. van Leer, Towards the ultimate conservative difference scheme, v. a second order sequel to godunov's method., J. Com. Phys. 32 (1979) $101-136$.

[9] R. Loubère, J.P. Braeunig, J.M. Ghidaglia, A totally eulerian finite volume solver for multi-material fluid flows: Enhanced natural interface positioning (enip), European Journal of Mechanics - B/Fluids 31 (2012) $1-11$.

[10] P.H. Maire, A high-order cell-centered lagrangian scheme for compressible fluid flows in two-dimensional cylindrical geometry, Journal of Computational Physics 228 (2009) 6882 - 6915.

[11] P. Roe, Upwind differencing schemes for hyperbolic conservation laws with source terms, in: C. Carasso, D. Serre, P.A. Raviart (Eds.), Nonlinear Hyperbolic Problems, volume 1270 of Lecture Notes in Mathematics, Springer Berlin / Heidelberg, 1987, pp. 41-51. 10.1007/BFb0078316.

[12] P.L. Roe, Approximate Riemann solvers, parameter vectors, and difference schemes, J. Comput. Phys. 43 (1981) 357-372.

[13] G. Strang, On the construction and comparison of difference schemes., SIAM Journal on Numerical Analysis 5 (1968) 506517. 